\title{
Análise do perfil do voluntariado para o manejo ecoturístico da Trilha Transcarioca na cidade do Rio de Janeiro
}

\author{
Analysis of the volunteer profile for the ecotourism \\ management of the Transcarioca Trail in the city of Rio de \\ Janeiro (RJ, Brazil)
}

\author{
Vivian Castilho da Costa, Thiago Martins Silva, \\ Ana Danielle Popescu Rogumbaun
}

\begin{abstract}
RESUMO: As trilhas servem como elo entre as pessoas e a natureza, servem também como vetores de diversos desequilíbrios ambientais, tais como introdução e propagação de espécies vegetais exóticas, pisoteio na vegetação, exposição, compactação e erosão do solo. A compactação tem desdobramentos, pois afeta o desenvolvimento de raízes, da vegetação, interfere nas taxas de infiltração hídrica, além de favorecer processos erosivos. Quando tais fenômenos se intensificam, passam a ser um sério problema gerencial para os administradores de Unidades de Conservação (UCs) no Brasil. O objetivo do presente estudo é o entendimento sobre o papel do voluntariado no manejo da Trilha Transcarioca, situada na cidade do Rio de Janeiro, e as possíveis mudanças no ecossistema local. O desafio de manter a Trilha Transcarioca é tornar o seu público frequentador em ator ativo na sua conservação e manutenção, subsidiando os trabalhos de estruturação e gerenciamento. A partir de estudos sobre o conselho consultivo, seus gestores e sua relação com os agentes sociais (governamentais e iniciativas privadas), buscou-se também a discussão sobre os impactos ambientais e territoriais (tanto os positivos quanto os negativos), para assegurar que o uso público aconteça sem comprometer a integridade da biodiversidade do local. Para a viabilidade do estudo, foi escolhido o método da pesquisa qualitativa do tipo exploratória e descritiva para realizar um inventário sobre o perfil do voluntariado e entender qual o seu papel na conservação da Trilha. A pesquisa concentrou o estudo em um trecho específico da Trilha Transcarioca no interior do Parque Nacional da Tijuca (município do Rio de Janeiro) - Trecho 13 - Portão da Floresta x Cova da Onça. Para a obtenção dos dados apresentados, utilizaram-se coletas de campo a partir de reuniões com a gestão da UC, com os adotantes da trilha e realização de questionários com os caminhantes e os voluntários de ações de manejo e conservação da Trilha Transcarioca, no período estabelecido entre o ano de 2016 até o primeiro semestre de 2018. Dessa maneira, as matrizes deste trabalho servem de análise de diversos aspectos que envolvem o uso público em unidades de conservação, não só o turismo sustentável e sua aptidão econômica, como também o manejo ecossistêmico e a conservação de todo corredor ecológico, divulgação de catálogos de registro de espécies da fauna/flora, palestras, seminários, pesquisas, programas de voluntariado e aspectos de segurança pública em prol da Trilha Transcarioca.
\end{abstract}

PALAVRAS CHAVE: Trilha; Conservação; Território; Paisagem; Gestão; Voluntários. 


\section{ABSTRACT}

Trails serve as a link between people and nature. They also serve as vectors of various environmental imbalances, such as introduction and propagation of exotic plant species, trampling on vegetation, exposure, compaction and soil erosion. The compaction has unfolding, since it affects the development of roots, of vegetation, interferes in the rates of water infiltration, besides favoring erosive processes. When such phenomena intensify, they become a serious managerial problem for the administrators of protected areas in Brazil. The objective of the present study is to understand the role of volunteering in the management of the Transcarioca Trail, located in the city of Rio de Janeiro, and the possible changes in the local ecosystem. The challenge of maintaining the Transcarioca Trail is to make its public an active player in its conservation and maintenance, supporting the structuring and management work. Based on studies on consultant councils, managers and their relationship with social agents (governmental and privates initiatives), discussions were also made on environmental and territorial impacts (both positive and negative) to ensure that public use occurs without compromising the integrity of the site's biodiversity. For the feasibility of the study, the use of the qualitative research of the exploratory and descriptive type was chosen to make an inventory about the volunteer profile and to understand its role in the conservation of the Trail. The following research concentrated the study within a specific section of the Transcarioca Trail in the Tijuca National Park (Rio de Janeiro county) - Section 13 Forest Gate x Cova da Onça. In order to obtain the data presented, there were collected information of the conservation actions based in fieldwork and interviews with the management of PNT, and was applied questionnaires with adopters, walkers and volunteers in the period established between the year 2016 to the first semester of 2018. In this way, the matrices of this work will serve to analyze several aspects that involve the public use of protected areas, not only sustainable tourism as economic aptitude, but also the ecosystem management and conservation of every ecological corridor, species fauna / flora, lectures, seminars, research, volunteering program and aspects of public safety in favor of the Transcarioca Trail.

KEYWORDS: Trail; Conservation; Territory; Landscape; Management; Volunteers.

\section{Introdução}

Tendo em vista a degradação recorrente dos sistemas ambientais brasileiros, o estabelecimento da fragmentação florestal ocorre quando um ecossistema é subdividido pela ação humana ou mesmo perturbações naturais, como o fogo, resultando em uma paisagem na qual permanecem alguns fragmentos de cobertura vegetal original inserido em uma matriz com características totalmente diferentes (KINDEL, 2001).

Dentro dessa lógica, a Mata Atlântica apresenta-se como de suma importância para preservação da biodiversidade, devido à alta diversidade biológica aliada a altas taxas de endemismo (ZAÚ, 1998). Este bioma além de possuir um pouco mais de $12 \%$ de sua área original ainda preservada, segundo dados da ONG SOS Mata Atlântica (2019), tem na sua localização e arranjo espacial, motivos de preocupação para conservação de sua biodiversidade, uma vez que, seu arranjo altamente fragmentado e sua localização, principalmente na região Sudeste e próxima a zona costeira 
(regiões caracterizadas pela maior ocupação humana), favorecem sua destruição. Por isso é importante entender este processo com suas relações espaciais em áreas de proteção como na formulação de formas de manejo e o uso sustentável de suas áreas.

Nesse sentido, o estudo sobre trilhas possibilitou pensar que estes caminhos são um instrumento de deslocamento dentro desses espaços (unidades de conservação da natureza). Entretanto, ao longo do tempo, houve uma mudança desta função, ou seja, de um simples meio de deslocamento, foram dadas às trilhas novas funções, tais como um meio de se entrar em contato direto com a natureza, conectando os visitantes com a paisagem (TUAN, 1980 e ROCHA, 2008) criando um novo turista ambientalmente sensibilizado, provocando mudanças de comportamento, tornando-o ator ativo nas tarefas de conservação, trazendo sensação de pertencimento. Quando determinado trecho de uma trilha se torna referência em visitação, as ações de manejo tornam-se frequentes, melhorando seu planejamento, tornando-se menos impactante (FIGUEIRÓ \& COELHO NETTO, 2009).

O trânsito de pessoas nessas áreas de conservação ocorre, em sua maioria, através de trilhas existentes para esse fim. No entanto, assim como as trilhas servem como elo entre as pessoas e a natureza, servem também como vetores de diversos desequilíbrios ambientais, tais como introdução e propagação de espécies vegetais exóticas, pisoteio na vegetação, exposição, compactação e erosão do solo (BARROS \& DINES, 2000). A compactação tem desdobramentos, pois afeta o desenvolvimento de raízes, da vegetação, interfere nas taxas de infiltração hídrica, além de favorecer processos erosivos (HAMMITT \& COLE, 1998). Quando tais fenômenos se intensificam, passam a ser um sério problema gerencial para os administradores das Unidades de Conservação (UC).

Para a execução de diagnósticos de integridade ecológica de trilhas faz-se necessário à procura por indicadores no ambiente que permitam uma avaliação de todo o sistema, considerando, suas interações espaciais. Com essa definição, o trabalho sobre a Trilha Transcarioca foi inicialmente idealizado por Pedro da Cunha e Menezes, em seu livro Transcarioca: todos os passos de um sonho (2000), respaldada em diversos exemplos bem sucedidos de trilhas de longo curso, tais como a Appalachian Trail (EUA), Huella Andina (Argentina), Hoerikwaggo Trail (África do Sul) e Te Araroa Trail (Nova Zelândia).

O objetivo principal da presente pesquisa é estudar os conflitos e as alterações do projeto de voluntariado da trilha Transcarioca (no trecho 13 Portão da Floresta x Cova da Onça do Parque Nacional da Tijuca - PNT, na cidade do Rio de Janeiro), caracterizando as relações existentes entre os atores envolvidos, assim como o papel dos seus gestores públicos, caminhantes e adotantes, buscando informações sobre como funcionam essas governanças e desafios que podem alterar a estrutura das gestões nas UC e suas transformações no espaço geográfico. 
Como objetivos específicos, o trabalho pretende oferecer condições que permitam fazer balanço dos avanços e limites de políticas de gestão pública voltadas à visitação em unidades de conservação, possibilitando a construção de conhecimento crítico por meio de troca das experiências e atividades em trilhas ecoturísticas, a exemplo da Trilha Transcarioca; a avaliação de levantamento de dados com o uso público no PNT (ICMBIOMMA, 2008) e suas características socioeconômicas na prática do ecoturismo, intentando o desenvolvimento sustentável e a proteção do meio físico e sociocultural.

\section{Localização e caracterização da área de estudo}

O percurso da Trilha Transcarioca tem um total de $180 \mathrm{~km}$ no interior do Parque Nacional da Tijuca. O Setor Floresta da Tijuca - Trecho 13 - Área do Portão da Floresta x Cova da Onça, tem a média de $5 \mathrm{~km}$ de extensão (Figura 1 e 2).

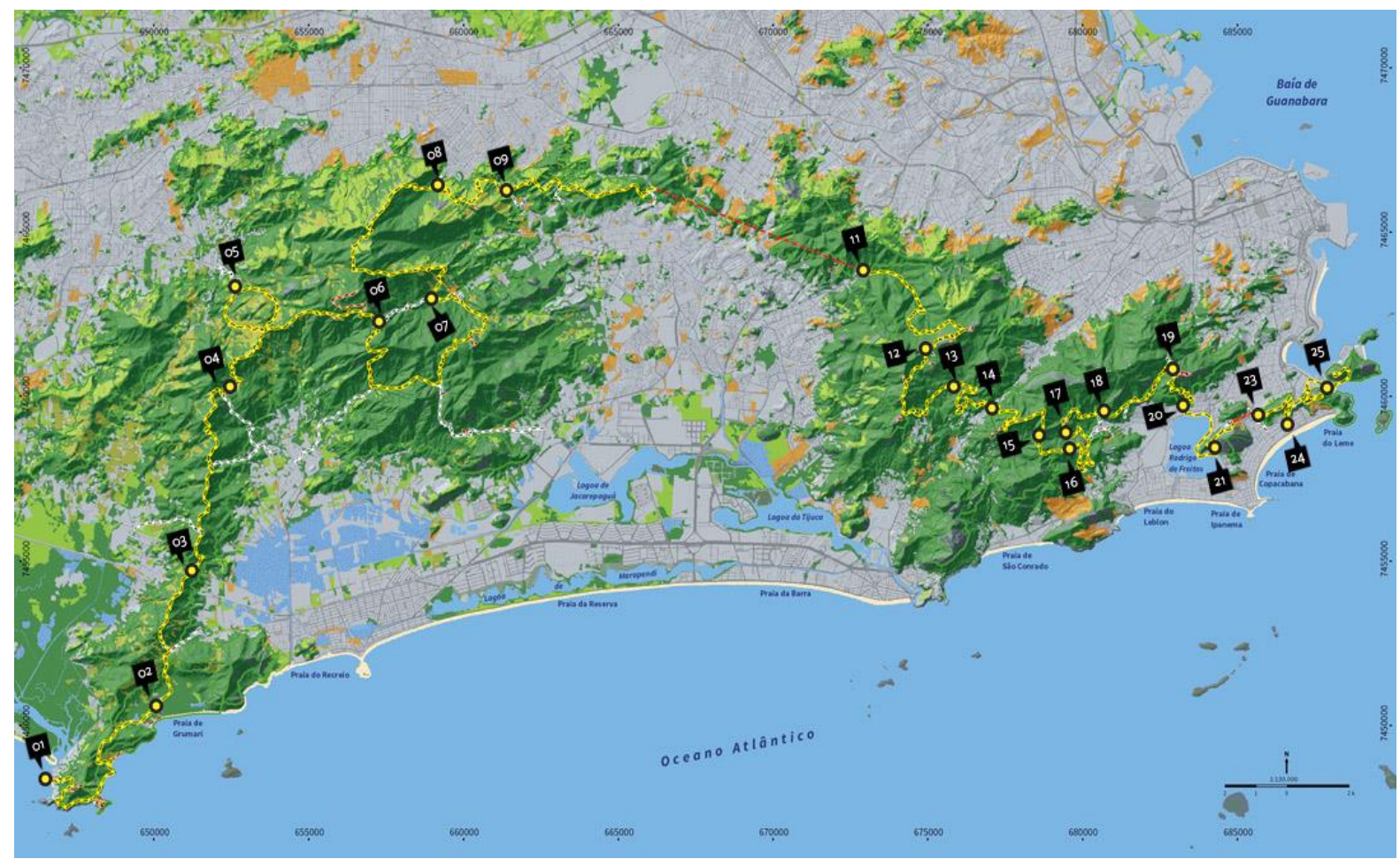

Figura 1: Localização da Trilha Transcarioca com a numeração dos seus 25 trechos (cidade do Rio de Janeiro, maciço da Pedra Branca a esquerda e maciço da Tijuca a direita).

Figure 1: Trail with the numbering of its 25 sections (Rio de Janeiro city, Pedra Branca massif on the left and Tijuca massif on the right).

Fonte: site da Trilha Transcarioca - <http://trilhatranscarioca.com.br/>.

Source: Transcarioca Trail website - <http://trilhatranscarioca.com.br/> 


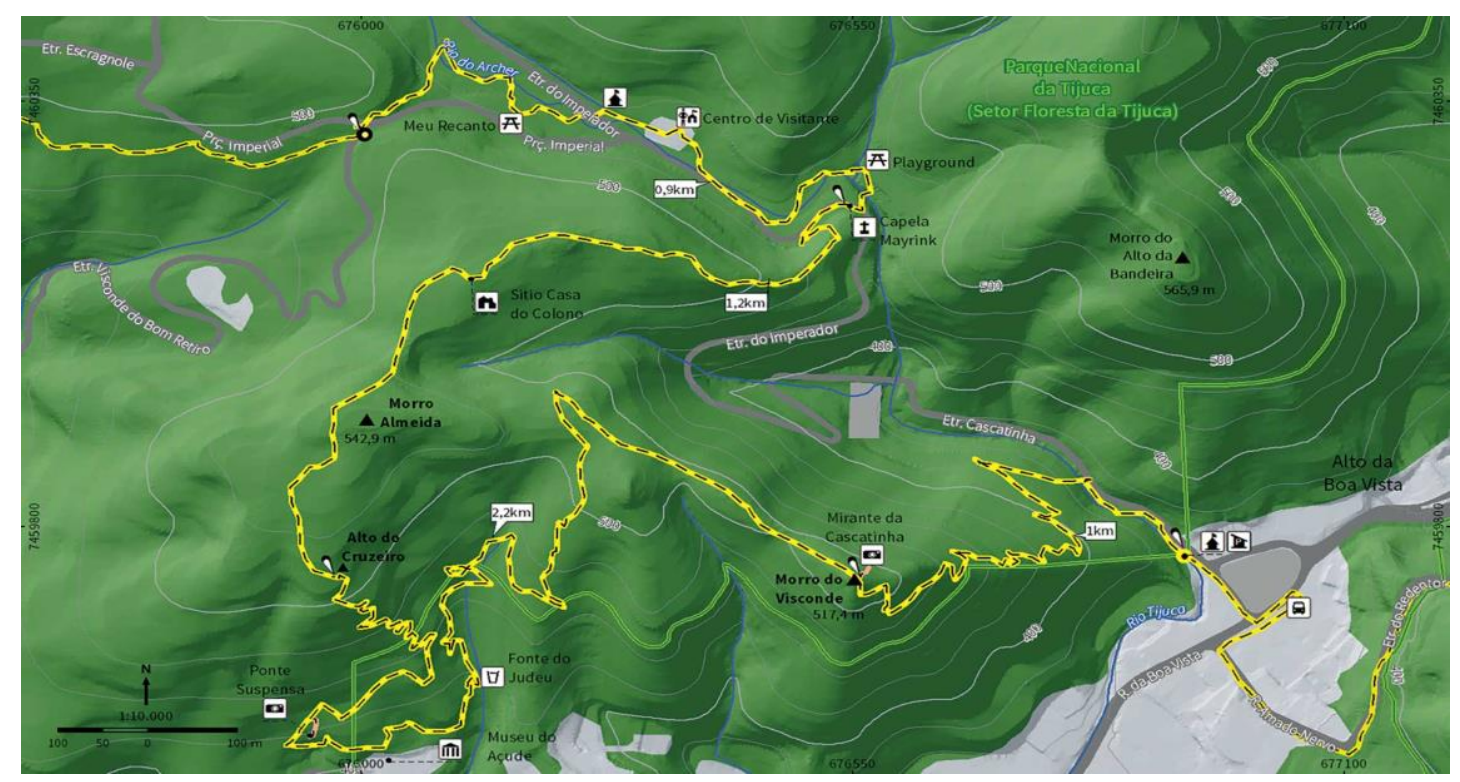

Figura 2: Detalhe do trecho 13 da Trilha Transcarioca no setor Floresta da Tijuca (PNT).

Figure 2: Detail of section 13 of the Transcarioca Trail in the Tijuca Forest (PNT) sector.

Fonte: site da Trilha Transcarioca - http://trilhatranscarioca.com.br/.

Source: Transcarioca Trail website - $\underline{\text { http://trilhatranscarioca.com.br/. }}$

Além de gerar emprego e renda dentro dos princípios norteadores do desenvolvimento sustentável, o propósito é que a Trilha Transcarioca siga o exemplo das trilhas de longo percurso estrangeiras, que geraram um incremento na visitação e diversas melhorias na gestão das áreas protegidas que a compõe. Os idealizadores da Trilha Transcarioca pretendem com o projeto de voluntariado proporcionar melhorias ambientais para a cidade, como a criação de um corredor florestal entre os maciços da Tijuca e da Pedra Branca e a unificação no manejo das unidades de conservação no Mosaico Carioca (ICMBio, 2011). O público frequentador das unidades de conservação envolvidas na Trilha Transcarioca é convidado a se inscrever no projeto de voluntariado com o intuito de contribuir para a conservação e manutenção da trilha e do corredor verde existente no Mosaico Carioca.

O trecho 13 da Transcarioca está localizado na entrada do portão principal da Floresta da Tijuca e tem diversos atrativos e possibilidades de integração com outras trilhas do percurso no interior do Parque Nacional da Tijuca, tais como: a Cascatinha Taunay, o Mirante da Cascatinha, o Centro de Visitantes do Parque Nacional da Tijuca, o Alto do Cruzeiro, o Museu do Açude, a Capela Mayrink, entre os principais.

Em 2015 foi fundada a Associação do Movimento Trilha Transcarioca (AMTT), fazendo desse projeto uma iniciativa que congrega pessoas e instituições da sociedade civil, através de um ofício de compromisso para a implantação da trilha, sua sinalização, manejo, divulgação e uso público qualificado. A AMTT tem caráter voluntário e colaborativo, de ordem pessoal ou institucional, e possui um sistema de governança próprio, com um grupo de coordenadores temáticos e um coordenador-geral, além de conselho 
próprio com diversas funções. Sua criação decorreu justamente da necessidade de organizar a interlocução entre voluntários, adotantes e colaboradores, com os gestores das unidades de conservação por onde a trilha passa, tanto individualmente (com os gestores/chefes das unidades de conservação) quanto institucionalmente (com os órgãos públicos municipais, estaduais e federais responsáveis).

A Trilha Transcarioca, reconhecida pelo Decreto Municipal № 43272 de 6 de Junho de 2017, interliga nove unidades de conservação de proteção integral de importância ecológica estratégica para a cidade como o Parque Estadual da Pedra Branca, Parque Natural Municipal de Grumari, Monumento Natural Municipal dos Morros do Pão de Açúcar e da Urca, Parque Natural Municipal da Cidade, Parque Natural Municipal da Catacumba, Parque Natural Municipal Fonte da Saudade, Parque Natural Municipal José Guilherme Merquior, Parque Natural Municipal da Paisagem Carioca, que permitem ainda o acesso a áreas protegidas como o Sítio Burle Max, o Parque Estadual da Chacrinha, o Museu do Açude e o Jardim Botânico do Rio de Janeiro.

A integração cria a ideia de um projeto que engloba Unidades de Conservação de várias categorias e esferas municipais, estaduais e federais, a exemplo do Parque Nacional da Tijuca, que corta áreas da zona sul e norte da cidade do Rio de Janeiro, onde se encontra o trecho 13, especificado no presente estudo como recorte espacial de análise.

\section{O que é o projeto de adotantes (voluntários) da Trilha Transcarioca?}

Além dos 1000 voluntários que já contribuíram para a implantação da trilha, existem instituições que ajudam eventualmente ou adotam trechos específicos da Transcarioca. Conforme afirma o coordenador do voluntariado Horácio Ragucci, na abertura oficial da Trilha, o local necessitou dos chamados adotantes de trechos dentro de seu percurso, visando colaborar com as unidades de conservação do Mosaico Carioca na sinalização e manutenção periódica da trilha, além de trabalhar em ações administrativas, de comunicação e outras tarefas necessárias a manutenção da Trilha. Calcula-se que mais de 10.000 horas de trabalho voluntário foram doados à Trilha Transcarioca somente em 2015. Esse fato confirma a importância e implicação da população com a manutenção e existência do projeto.

Para fins de sua manutenção e de trabalhos voluntários, a Trilha Transcarioca está dividida em 26 trechos, sendo oficialmente 25 trechos utilizados até o momento, dos quais atualmente 23 têm adotantes voluntários permanentes, sendo os demais locais com adoções em processo de transição e análise, mas com projetos envolvendo o voluntariado e os adotantes. A adoção é feita por meio de um termo assinado pelo adotante, pelos Amigos da Trilha Transcarioca e pela Unidade de Conservação em que o trecho adotado está inserido. Ao assinar o termo de adoção de um trecho, o adotante se responsabiliza por fazer ações periódicas de manutenção (idealmente pelo menos uma por mês), verificando a 
sinalização, repondo as setas indicativas e orientadoras com a logomarca da Transcarioca (pegadas) que estiverem faltando, desobstruindo a trilha sempre que houver galhos ou árvores caídas e bloqueando o caminho (Figura 3), retirando lixo que porventura estiver no leito da trilha e fazendo abordagens educativas com visitantes de acordo com o movimento da Trilha Transcarioca desde $2016 \mathrm{com}$ a implantação do projeto. Dependendo do número de voluntários que cada adotante tiver em seu grupo, a ação de manutenção poderá ser semanal ou até diária.

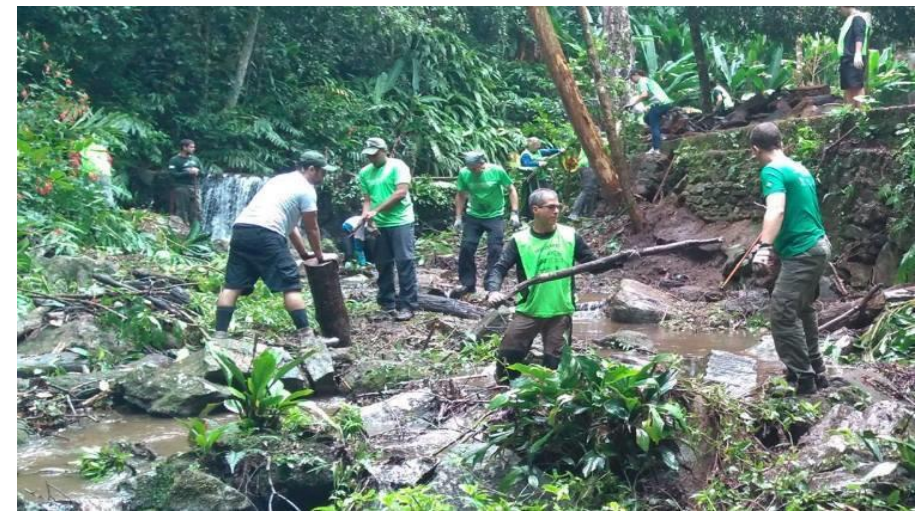

Figura 3: Trabalho Voluntário de manutenção no Trecho 13 da Trilha Transcarioca no PARNA Tijuca - Setor A, em 2015. Fonte: Foto dos Autores.

Figure 3: Voluntary maintenance work on the Transcarioca Trail 13 in PARNA Tijuca Sector A, in 2015. Source: Photo by the Authors.

\section{Materiais e Métodos}

Para a viabilidade do estudo, foi escolhido o uso da pesquisa qualitativa do tipo exploratória e descritiva. A pesquisa qualitativa, de acordo com Richardson et. al. (2007, p. 90), "[...] pode ser caracterizada como a tentativa de uma compreensão detalhada dos significados e características situacionais apresentadas pelos entrevistados, em lugar da produção de medidas quantitativas de características ou comportamentos".

A pesquisa do tipo exploratória é realizada por meio de aplicação de entrevista semiestruturada, a partir de questões abertas e fechadas, via contato direto. Com base nesse método, foi elaborado um questionário com perguntas com o objetivo de avaliar as percepções, entendimentos das ações estabelecidas pelo programa de voluntariado, noção espacial das ações, conhecimento do bioma, estruturas usadas no manejo e conservação da trilha, tipos de ações existentes no período da aplicação do questionário, o contato com a natureza pelos adotantes do trecho, voluntários e gestores para o levantamento de uma base de dados sobre o projeto de voluntariado e a integração das gestões dentro dos trechos adotados.

As coletas de dados foram realizadas a partir das reuniões de gestão dos adotantes e trabalhos de campo com questionários no período estabelecido de 2016 até o primeiro semestre de 2018, em pontos específicos onde se obtém maior fluxo de pessoas no trecho, de acordo com dados coletados com o próprio Parque em sua organização e gestão. 
No trecho 13 da Trilha Transcarioca foram aplicados questionários (Figura 4) para um total de 150 pessoas; entre caminhantes, gestores e voluntários. No período estabelecido da presente pesquisa foram entrevistados 50 voluntários que desenvolveram 14 ações no trecho 13.

\section{LAGEPRO-}

Colaboradores: Thiago Martins( PPGEO-UER) e Ana Popescu (Graduanda-Biologia-UNTVERSO)

\section{FICHA DE CAMPO

VOLUNTARIADO :

[Antes de (re)iniciar o trabalho de campor fotografar o equipamento utilizado e a equipe no local para anexar ao relatório final deste campo; verificar se o equipamento esta seguro] INFORMAÇÕES GERAIS: Campo $\mathrm{n}^{\circ}$.

IRILHA TRANSCARIOCA TRECHO 13 - PARQUE NACIONAL DA TIUUCA DATA: DIA DA SEMANA OBJE TOS / FERRAMENTAS UTILIZADOS (AS) NES TE CAMPO (LIS TAGEMD:

Atividades realizadas no dia:

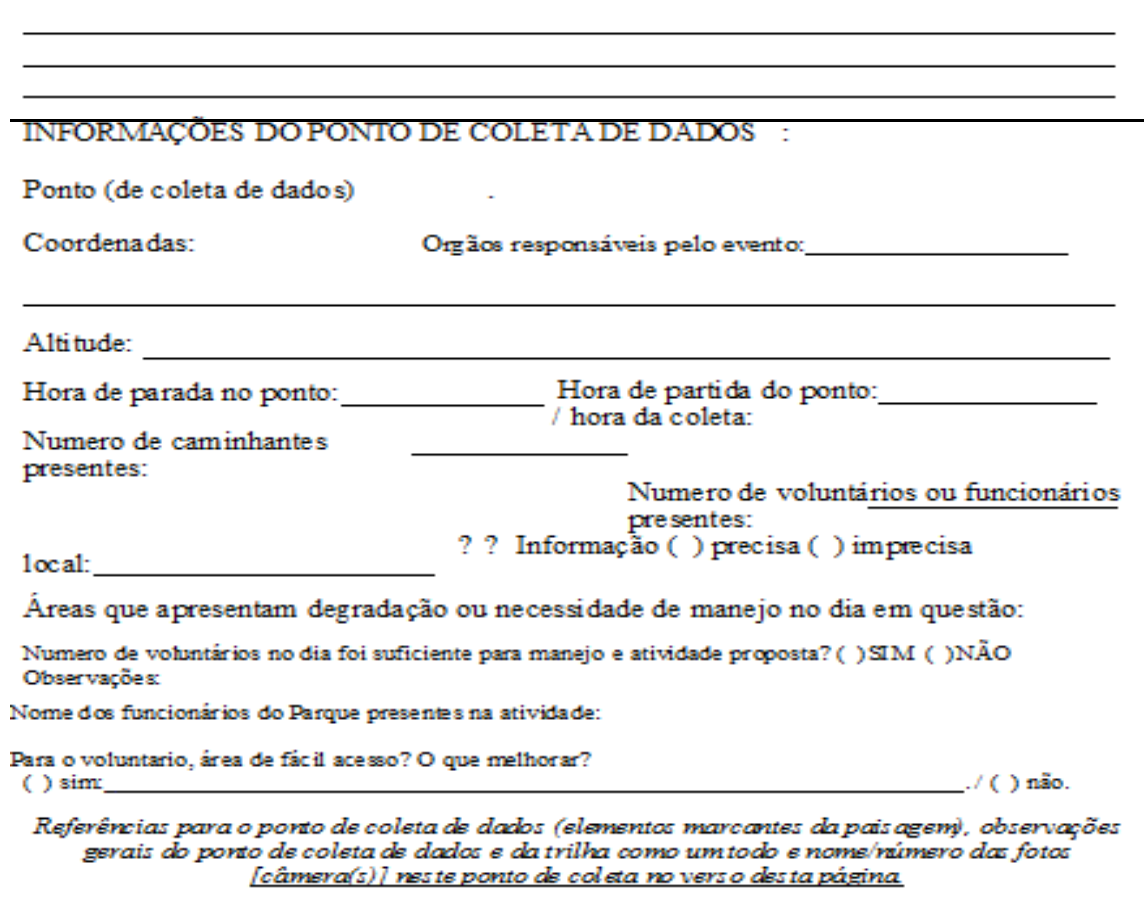

Figura 4: Exemplo da primeira parte do questionário utilizado no trecho 13.

Figure 4: Example of the first part of the questionnaire used in excerpt 13.

Fonte: Elaborado por Silva e Popescu- (2016 - 2018).

Source: Prepared by Silva and Popescu- (2016 - 2018).

No intuito de melhor atender aos objetivos da presente pesquisa na análise do perfil dos visitantes engajados nos projetos de voluntariados no PNT, foram criadas perguntas relacionadas com os seguintes questionamentos: a) Qual o perfil socioeconômico dos frequentadores; b) Se 
existem práticas deliberadas dos grupos de caminhantes para ocorrer trocas de saberes e quais seriam estas; c) Se o entrevistado possui noção sobre localização geográfica e sobre a importância histórica da área; d) Qual o grau escolar e a idade das pessoas envolvidas nas entrevistas; e) Qual a área de moradia do voluntário e se esta é próximo do PNT; f) Qual a real percepção sobre a importância floresta e quais os elementos marcantes da paisagem como atrativos turísticos.

Os resultados foram tabulados e analisados usando o recurso computacional do Microsoft Office Excel 2017.

\section{Resultados}

\section{Dados analisados a partir dos questionários}

A partir dos resultados tabulados dos questionários aplicados, podese constatar que a maioria dos voluntários ( $85 \%$ ) é maior de idade (18 anos), sendo que a metade dos entrevistados possui nível superior. Levando em conta os estudos de Malta e Costa (2009), o nível cultural dos indivíduos deve ser um elemento balizador nos programas de Educação e Interpretação Ambiental em unidades de conservação.

Dentre os voluntários atuantes, $74 \%$ é de moradores de áreas próximas ao local, tendo como diferença o perfil dos caminhantes, onde 0 total de $92 \%$ mora em bairros distantes (com 2 horas de trajeto para o local) ou até mesmo outras cidades da região metropolitana. Dentro do perfil estabelecido dos gestores entrevistados no Trecho 13 a pesquisa revelou que os funcionários do PNT que atuam com o voluntariado possuem uma estabilidade no local, sendo que muitos decidem seguir carreiras relacionadas com o meio ambiente no nível superior.

A partir dos dados coletados sobre a percepção ambiental é possível mensurar que a água apareceu como um tema relevante, fazendo com que $66 \%$ de todos os entrevistados elegessem a Cascatinha Taunay e os córregos visualizados na trilha como pontos de atrativos turísticos preferidos para contemplação. Dentre os monumentos histórico/artísticos presentes na Trilha dos Estudantes (Trecho 13), os bancos de alvenaria com azulejos portugueses/franceses e as banheiras em mármore Carrara foram apontados por $65 \%$ do total de entrevistados como principais destaques de atrativos turísticos. Os questionários revelaram ainda que 90\% dos caminhantes de trilhas consideram importante o contato com a natureza, $80 \%$ pretendem voltar ou já foram mais de 1 vez ao Parque Nacional da Tijuca; $57 \%$ dos caminhantes trabalharam anteriormente com projetos de voluntariado.

Dentre todos os entrevistados, $98 \%$ consideraram importante 0 projeto para a manutenção das trilhas, sendo que consideram o trecho 13 como essencial para a Floresta da Tijuca, devido a sua localização na entrada da floresta, permitindo, com a sua conservação, o melhor acesso aos atrativos como o Pico da Tijuca e do Papagaio. 
O Parque Nacional da Tijuca, dentro do projeto da Trilha Transcarioca vem sendo considerado pelos seus idealizadores como um modelo bemsucedido na linha inicial do voluntariado, contando com uma série de ações organizadas pela estrutura do Parque. Contudo, tendo em vista a relação que esse corredor verde pretende com as suas ações, ainda existe diversos pontos a tratar, incluindo os recursos e a gestão compartilhada e interligada não só com o Parque, como também com outras áreas de conservação da Trilha e com outras unidades de conservação na região metropolitana do Rio de Janeiro.

\section{Força do voluntariado dentro das unidades de conservação}

Como afirma Anderson Ribeiro, um dos gestores da Trilha Transcarioca, as ações recentes e mudanças de protocolos de conservação com o voluntariado vem ganhando força dentro das unidades de conservação e já faz parte das ações estratégicas na gestão dessas unidades.

Dentro do Brasil há muitas áreas protegidas por lei, porém com grande escassez de funcionários em todos os segmentos, sejam esses analistas ambientais, guardas parque, monitores, pessoas responsáveis pela limpeza, entre outros. O voluntariado acaba por auxiliar para que não haja sobrecarga de atividades, "somando forças". Há unidades onde é possível encontrar mais voluntários do que funcionários e todos atuantes.

A partir dos resultados da pesquisa, é possível estabelecer que o Parque Nacional da Tijuca com o projeto de voluntariado vem contando com uma série de ações organizadas pela estrutura do Parque e, uma delas, é a adoção de trilhas, como efetivação da gestão compartilhada. É o voluntário que passa por uma capacitação dentro da unidade, assinando um termo de responsabilidade e passa a realizar mutirões com ações de manejo, de sinalização e de educação ambiental. Através de uma iniciativa voluntária, as trilhas de longo percurso vêm contribuindo bastante com a conservação dessas áreas protegidas, pois fortalece os corredores verdes, fomenta 0 turismo, recupera a história, proporciona estudos, pesquisas, educação ambiental, além de gerar emprego e renda em vários segmentos, ganhando assim, o reconhecimento devido através de seus resultados.

No Rio de Janeiro, a Trilha Transcarioca chegou com esse modelo piloto e hoje é a precursora e referência no Brasil, inspirando projetos como o caminho da Mata Atlântica e o desenvolvimento de uma trilha do Oiapoque $x$ Chuí, com potencial de ser, não apenas maior em tamanho, mas o dobro do tempo de percurso da Appalachian Trail.

A Trilha Transcarioca recentemente ganhou prêmios em grandes eventos de montanhismo e é assunto de uma série de pesquisas acadêmicas. Destaque-se, ainda, que a Trilha possui um aplicativo exclusivo para mobiles e foi roteiro oficial durante os jogos olímpicos através do programa "Passaporte Verde", que divulgava atividades sustentáveis na cidade do Rio de Janeiro. 


\section{Considerações Finais}

Com a pesquisa do perfil do voluntariado no trecho 13 do PNT foi possível identificar que o projeto vem conseguindo atender ao seu objetivo principal que é o de conservação de trilhas. Observou-se ainda que as tendências atuais dentro da prática do ecoturismo e abertura de trilhas ainda estão sendo disseminadas.

Essas tendências podem ser consideradas a partir de um reflexo do amadurecimento de projetos que visam a integração do uso público $x$ gestão, uma vez que cada vez mais a ideia de proteção e entendimento sobre o ambiente natural da cidade do Rio de Janeiro vem necessitando de estímulos com a inserção da educação ambiental que ainda não faz parte do cotidiano do projeto de voluntariado na Trilha Transcarioca. A inserção de capacitações em manejo de trilhas vem trazendo, no entanto, agentes ativos na conservação, caminhando para práticas utilizadas pelos gestores, quando pensam na manutenção dos corredores verdes na cidade do Rio de Janeiro.

Buscou-se, com a pesquisa, realizar uma discussão sobre o papel exercido pela percepção (GUIMARÃES, 2004) dos participantes do voluntariado em relação às paisagens naturais na Trilha Transcarioca. Pôde-se concluir que, embora em parte não se possa negar o papel das diferentes formas de mídia, incluindo as mídias sociais no incentivo ao crescimento do projeto, a necessidade humana pela natureza parece ser muito mais frequente e constitui um incentivo muito mais forte para comprovar que as paisagens têm de fato, um significado afetivo entre os entrevistados, pois estes querem permanecer como voluntários pela vontade de contribuir com a conservação do ambiente e, assim, fazendo parte de ações de manejo da trilha.

É importante ressaltar que a pesquisa está em andamento com novas entrevistas visando ampliar as análises sobre o projeto de voluntariado em todo o trecho 13 e como estudo futuro, planeja-se realizar comparação com outros trechos da Trilha Transcarioca no PNT. Serão inventariados outros aspectos a exemplo do planejamento da sinalização da trilha e aspectos sobre os riscos ambientais associados à visitação.

\section{Referências}

BARROS, M. I. A; DINES, M. Mínimo Impacto em Áreas Naturais: uma mudança de atitude. In: SERRANO, C. (Org.). A educação pelas pedras: Ecoturismo e educação ambiental. São Paulo: Chronos, 2000. p. 47-84.

Decreto Municipal № 43272, de 6 de Junho de 2017. Prefeitura da Cidade do Rio de Janeiro. Reconhece e denomina a Trilha Transcarioca. Disponível em: $\quad<$ https://leismunicipais.com.br/a/ri/r/rio-de-janeiro/decreto/2017/4328 143272/decreto-n-43272-2017-reconhece-e-denomina-a-trilha-transcarioca-eda-outras-providencias >. Acesso em: 30/06/2019. 
FIGUEIRÓ, A. S.; COELHO NETTO, A. L. Análise do impacto produzido por trilha sem áreas de borda florestal no maciço da Tijuca. Disponível $<$ www.scribd.com/doc/11566459/artigoadriano-severo-figueiro>

GUIMARÃES, S. T. L. Dimensões da Percepção e Interpretação do Meio Ambiente:vislumbres e sensibilidades das vivências na natureza. Ciência \&Tecnologia, Rio Claro, vol.4, n. 1, p. 46-64, abril/2004.

HAMMITT, W.; COLE, D. N. Wildland Recreation: Ecology and Management. New York: John Wiley \& Sons, 1998.

ICMBIO - MMA. Plano de manejo do Parque Nacional da Tijuca, 2008. Disponível em: <http://www.icmbio.gov.br/portal/images/stories/docs-planosde-manejo/parna tijuca pm.pdf >. Acesso em: 30/06/2019.

ICMBIO. Portaria de reconhecimento do Mosaico Carioca, 2011. Disponível em: <http://www.icmbio.gov.br/portal/mosaicosecorredores ecologicos/moscaicos-reconhecidos-oficialmente/1860-mosaico-carioca28>. Acesso em: 30/06/2019.

KINDEL, A. A fragmentação real: Heterogeneidade de remanescentes florestais e valor indicador das formas de húmus. Tese (Doutorado em Geografia) - Rio de Janeiro - RJ, Universidade Federal do Rio de Janeiro UFRJ, 188p. 2001.

MALTA. R. R.; COSTA, N. M. C. Gestão do Uso Público em Unidade de Conservação: a visitação no Parque Nacional da Tijuca - RJ. Revista Brasileira de Ecoturismo, São Paulo, v.2, n.3, 2009, pp.273- 294.

MTT, Movimento Transcarioca. http://transcarioca.wikiparques.org/comoajudar/, 2016.

ROCHA, J. C. Diálogo entre as categorias da geografia: espaço, território, e paisagem. Dissertação de Mestrado em Geografia da Pontifícia Universidade Católica de São Paulo - PUC,2008. Disponível em: $<$ http://www.seer.ufu.br/index.php/caminhosdegeografia/article/view/15724/8 897>. Acesso em: 30/06/2019.

SOS Mata Atlântica. Atlas da Mata Atlântica: Dados mais recentes, 2019. Disponível em: <https://www.sosma.org.br/projeto/atlas-da-mata-atlantica/ dados-mais-recentes/>. Acesso em: 30/06/2019.

TUAN, Y. Topofilia. Um estudo da percepção, atitudes e valores do meio ambiente. Tradutora: Lívia de Oliveira. São Paulo/Rio de Janeiro: DIFEL, 1980.

ZAÚ, A. S. Fragmentação da Mata Atlântica: aspectos teóricos. Floresta e Ambiente. Vol.5(1):160-170, jan/dez. 1998. (Professor Mestre- UFRRJ). 
Vivian Castilho da Costa: Universidade do Estado do Rio de Janeiro, Rio de Janeiro, RJ, Brasil

E-mail: vivianuerj@gmail.com

Link para o currículo Lattes: http://lattes.cnpq.br/3181407490194397

Thiago Martins Silva: Universidade do Estado do Rio de Janeiro, Rio de Janeiro, RJ, Brasil

E-mail: silvamartinsthiagogeo@gmail.com

Link para o currículo Lattes: http://lattes.cnpq.br/8998747780890279

Ana Danielle Popescu Rogumbaun: Faculdade Universidade Salgado de Oliveira, Rio de Janeiro, RJ, Brasil

E-mail: ana_danielle96@hotmail.com

Link para o currículo Lattes: http://lattes.cnpq.br/3903704751980497

Data de submissão: 26 de março de 2019

Data de recebimento de correções: 21 de junho de 2019

Data do aceite: 21 de junho de 2019

Avaliado anonimamente 